
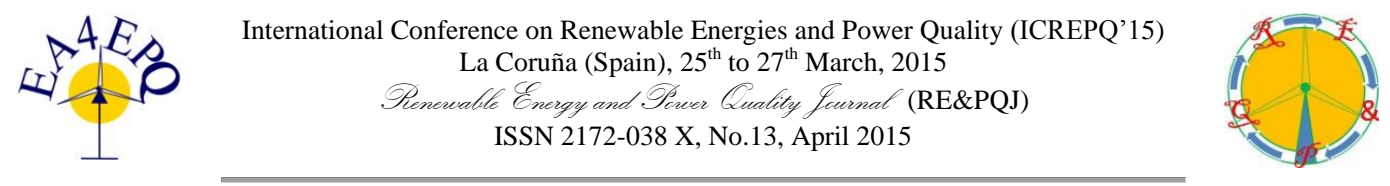

\title{
A system for visualization of power-quality and optimization of the charging behavior for electric vehicles
}

\author{
Y. Wang ${ }^{1}$, B. Schwake ${ }^{2}$, B. Fischer ${ }^{1}$ and P. Stroganov ${ }^{1}$ \\ ${ }^{1}$ Department of informatics \\ Clausthal University of Technology \\ Arnold-Sommerfeld-Str.1, 38678 Clausthal-Zellerfeld (Germany) \\ e-mail: yong.wang@tu-clausthal.de, benjamin.fischer@tu-clausthal.de, peter.stroganov@tu-clausthal.de \\ ${ }^{2}$ Institute of Electrical Power Engineering and Energy Systems \\ Clausthal University of Technology \\ Leibnizstraße 28, 38678 Clausthal-Zellerfeld (Germany) \\ email: benjamin.schwake@tu-clausthal.de
}

\begin{abstract}
Optimization of the dynamic behavior of the charging process for electric vehicles requires a centralized adjustment of control parameters. The development of suitable algorithms for the adjustment needs a tool to analyze and to visualize measured data from the environment of the charger. In this paper, we present that Power-Quality Map, a system to collect data and to show them on a map, which offers methods for analyzing those measured data. This paper describes the structure of the system, the software architecture and the functionality to visualize maps.
\end{abstract}

\section{Key words}

Power-Quality Map, electric vehicle, visualization tool, charging behavior

\section{Introduction}

The declining electricity generation capacity of large power plants demands the transition to a flexible electricity supply. The energy system is challenged to provide the accustomed stability in spite of rising residual load gradients. So far central services as inertia, primary reserve and secondary reserve were provided by large power plants. Now these services have to be adopted by distributed systems.

From this point of view growing electric mobility creates additional challenges but also holds chances for the energy system. On the one hand, a high power rating is needed to facilitate charging of electric vehicles in acceptable time; on the other hand, every electric vehicle makes a high-power, high-energy battery available. Assuming an appropriate control, these energy storages could be used to stabilize the grid.
In this context Clausthal University of Technology is working on grid-beneficial charging modes for electric vehicles with the ability to offer operating reserve via the vehicles batteries. Here investigations are centered on operating reserve in the transient and sub-transient time domain. The proposed approach uses a control-method which works on two different levels.

On the lower level, a locally closed control loop (Fig. 1) regulates the charger. It instantaneously reacts to fluctuations in grid voltage $U$ and frequency $f$. Therefor it utilizes the concept of the Virtual Synchronous Machine (VISMA) [1-3] and leans on measurements locally captured by the chargers sensors. The charging power will be adapted to the grid-state continuously.

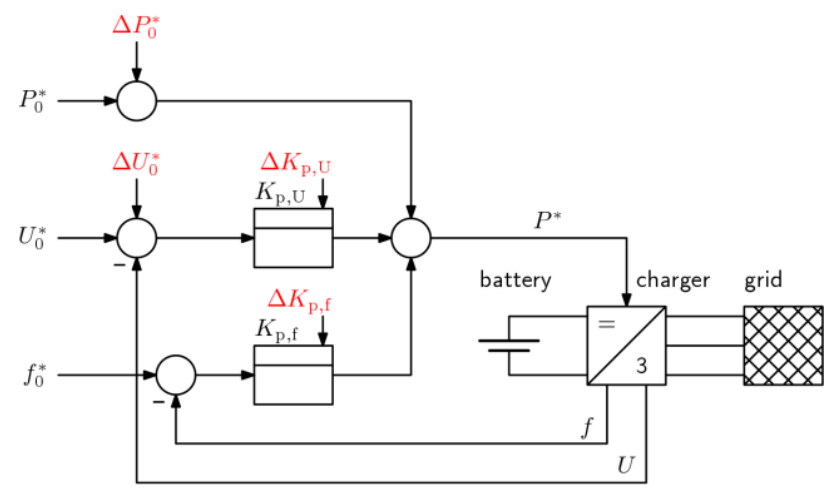

$P_{0}^{*}$ : nominal charging power

$U_{0}^{*}$ : nominal grid voltage $(230 \mathrm{~V})$

$f_{0}^{*}$ : nominal grid frequency $(50 \mathrm{~Hz})$

Fig. 1. Local control loop for electric vehicle charger

On the higher level the dynamic behavior of the charging method is optimized through a centralized adjustment of control-parameters $\Delta K_{p, U}$ and $\Delta K_{p, f}$ (Fig. 1). Therefor 
measurement values which are generated by the chargers and which are describing the local grid state for a certain instant of time are sent to a central server where they are stored and processed. Based on this data it is investigated whether it is possible to describe the grid state in certain areas and how the charging-parameters could be optimized for these areas. The developed algorithms will later be used to continuously optimize the parameter sets and distribute them to the electric vehicles in normal operation.

This paper describes the development of a time-dependent map of power-quality as a tool for analysis of grid-state. This so called "Power-Quality Map" is an essential component of the centralized data processing system. The aggregation of data from various sources and storage of those in a statistics-database together with the visualization via a map are important for the development of algorithms for continuous optimization of the chargingparameters of electric vehicles connected to this system.

\section{System structure}

This paper presents the concept of a centralized computing software system, which is responsible for data processing and preparation (Fig. 2). The System implements a Web Application Server, which runs on a Linux operating system in a Secure Cloud. The Secure Cloud ensures requirements of a high security level. When the amount of data is increasing the cloud system can increase its storage space easily.

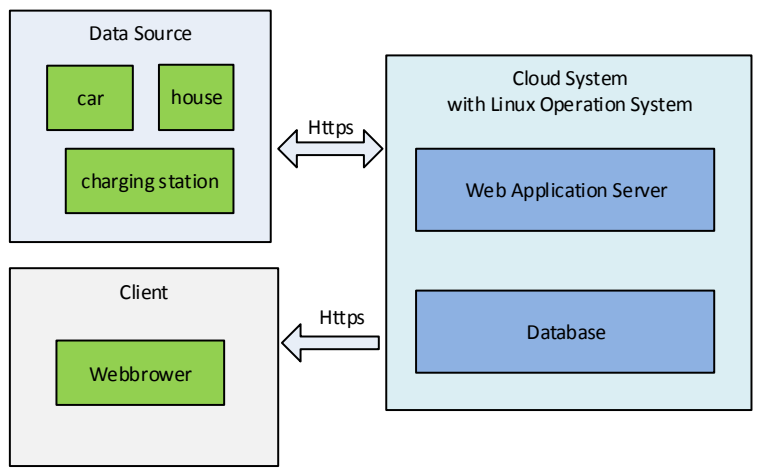

Fig 2. System structure of Power-Quality Map

The system can receive measured data from distributed devices. A device can be a telemetry device in an electric car, a smart-meter in a charging station or a household electricity meter with server function. In this paper, the main data comes from electric vehicles. The data transfer is secured by using HTTPs (Hypertext Transfer Protocol Secure) with SSL (Secure Sockets Layer). The measured data must contain the values for device-ID (car-ID or house-ID), timestamp, GPS position, voltages, currents and frequency. More information such as charging behavior and battery information can be received by the system too. That information is important for optimization of the charging behavior. All the data is stored to a SQL database via Web Server. One of the most important tasks of the system is to present the power-quality. The data about the power-quality are at first calculated and processed by the use of the web application server, then the processed data is presented on the map type that is previously selected by user in the web browser. To use such maps the user has to have appropriate authorization.

\section{Software architecture}

The system is built according to three-layer architecture [5] with using components (Fig. 3). With this architecture, the system can be easily extended in the future and further algorithms can be integrated into an existing software system using such architecture. The exchange of information from one layer to another layer or between two components is only possible by using interfaces. It is secure that developers can change the implementation of components without changing other parts of the system. The solution we recommend will be explained in detail in the following section.

\section{A. Persistent Layer}

In the persistent layer the data model is defined, which is used to access data in the database. The data model consists of two components: „ChargingStationRep“ and „BorderDataRep“. The first one is used to store measured data from measurement devices. The second one is used to store additional settings for visualization on maps. Component "ChargingStation" holds different data models in order to store data about status of the network, charging behavior and position of the device to present on the map; "BorderDataRep" stores the geographical borders.

\section{B. Logic Layer}

This layer is an application-logic layer. The functions in this layer have two important purposes: on the one hand, it can present the maps for the user; on the other hand, it sends and receives data from measuring devices. The "Map Manager" component is used to visualize the power-quality. To create a power-quality map this component should first call the functions from the component "Computing". Then, the function in the component "Computing" computes the data received from the Persistence layer by interface and sends it back. After that the results are sent to "Web Code Generator" component via "Map Manager" component. These results are written in a special script which allows data to be shown on the map. The algorithms for sequential optimization of the charging parameters for electric vehicles are planned in the component "Computing". The accumulated data is translated via component "DataTranslate" and stored in the database through the persistent layer interface. 


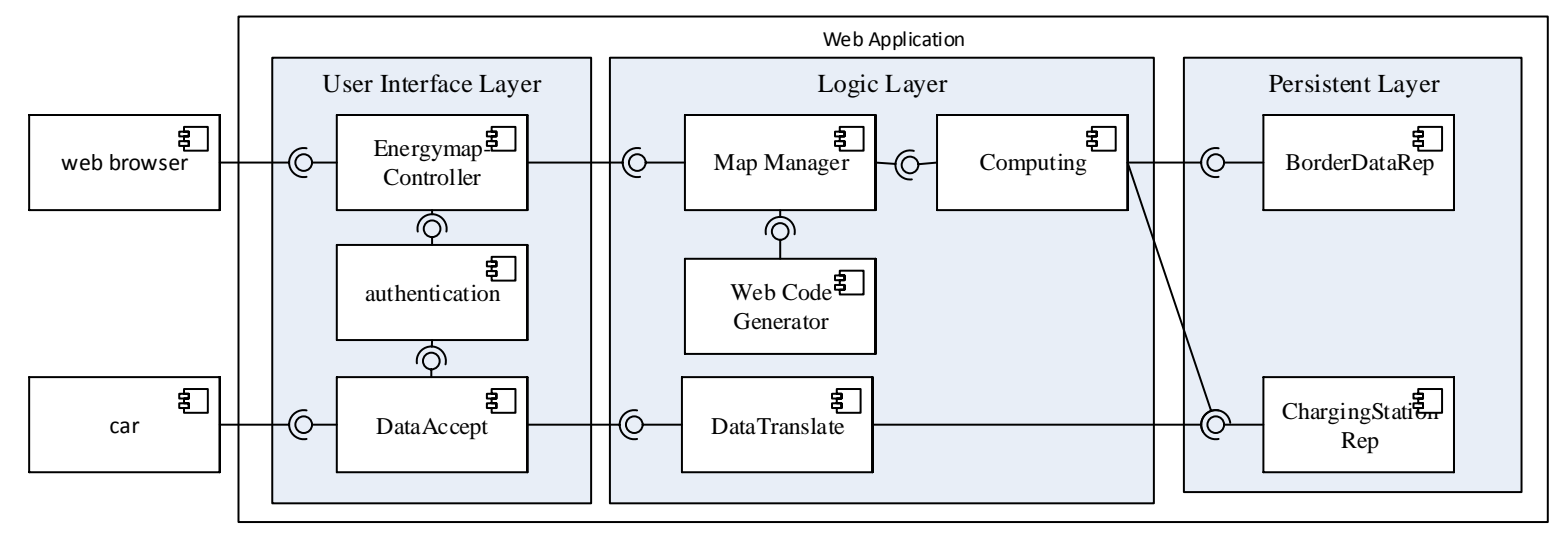

Fig. 3. Component diagram of the proposed software system

\section{User Interface Layer}

The Task to communicate with users and devices is realized in the user interface layer by using the MVC (Model View Controller) architecture. The component "EnergymapController" is used to send data and to receive data from web browsers. Data from electric vehicles can be stored in this software system using "DataAccept" and "DataTranslate" components in the persistent layer. Security methods are integrated into this layer by using the "Authentication" component in order to provide user authentication. By the help of these mechanisms, personal related information may be shown only if users have the appropriate authorization. Personal related data from any device are not stored without user's permission.

\section{Power-Quality Map}

The most important task of the project lies in visualizing the Power_Quality Map. The existing „Power Map“[4] shows the measured information and information only from device as landmarks. The measurement data are collected only from fixed devices. The proposed system can collect power_quality data from mobile and fixed devices. It is possible to show power_quality information in the required period of time and area with several presentation methods on the map. With these functions it reduces not only the time for analyzing the change of power_quality, it facilitates the work for testing algorithms for optimizing the charging parameters in field experiments.

The following three methods of representation are being developed, the so-called Points Map, Heat Map, Color Map and chart.

\section{A. Point Map}

Each position of a charging device is represented as a marker on the map. The positon of moveable devices is collected using GPS signal. While a device is not moving, it still can send different position values, because of the tolerance of GPS-signals. Therefore, this system has an algorithm to record the first GPS-position a device sends as a reference point. Then if the following recorded GPS signals read from the same GPS device are within an area of $2 \mathrm{~m}^{2}$ of the reference, the points are regarded at the same position. Otherwise, the GPS signal is stored with a new position and displayed as a new location on the map.

This system doesn't show realtime information on the map. The map only uses average voltage and current values in a user defined period of time. The average voltage for a position of a device defines the color of the marker (Fig. 4). With those markers users can quickly get an overview of power-quality in a certain area. After clicking on a marker, detail information will be shown on a label.

The original voltage that is collected from a single device can be presented as a waveform diagram. Through this program, the voltage profile over time of a point can be analyzed exactly.

Only legitimate users can use this map and chart because the map contains personal information.

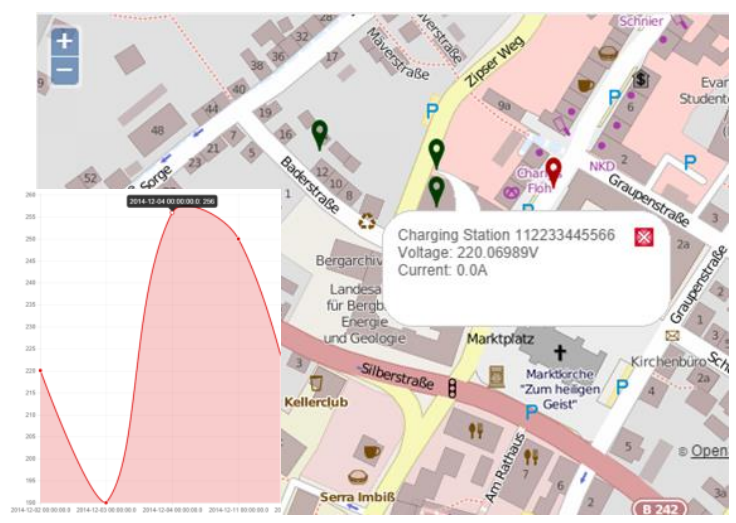

Fig.4. Point Map and waveform diagram.

\section{B. Heat Map}

The abovementioned Point Map is chosen in this work to observe the power-quality in a small area. If someone 
wants to analyze the power-quality in a bigger area, the system offers the Heat Map (Fig. 5). By using the Heat Map, users can find weak spots of the electric network very quickly. By changing the period of time, users can analyze the temporal changes of the electric network condition. Within this mode, the electric network is represented by the circles of different color and density. Circles containing more red than blue in the center represent more dense areas and also show deviations, where voltage is too high or too low. When zoomed in, the map shows areas where deviations from norm-values occur as more intense points with bigger radius. When zoomed out, the density of the network is shown and more used areas can be noticed.
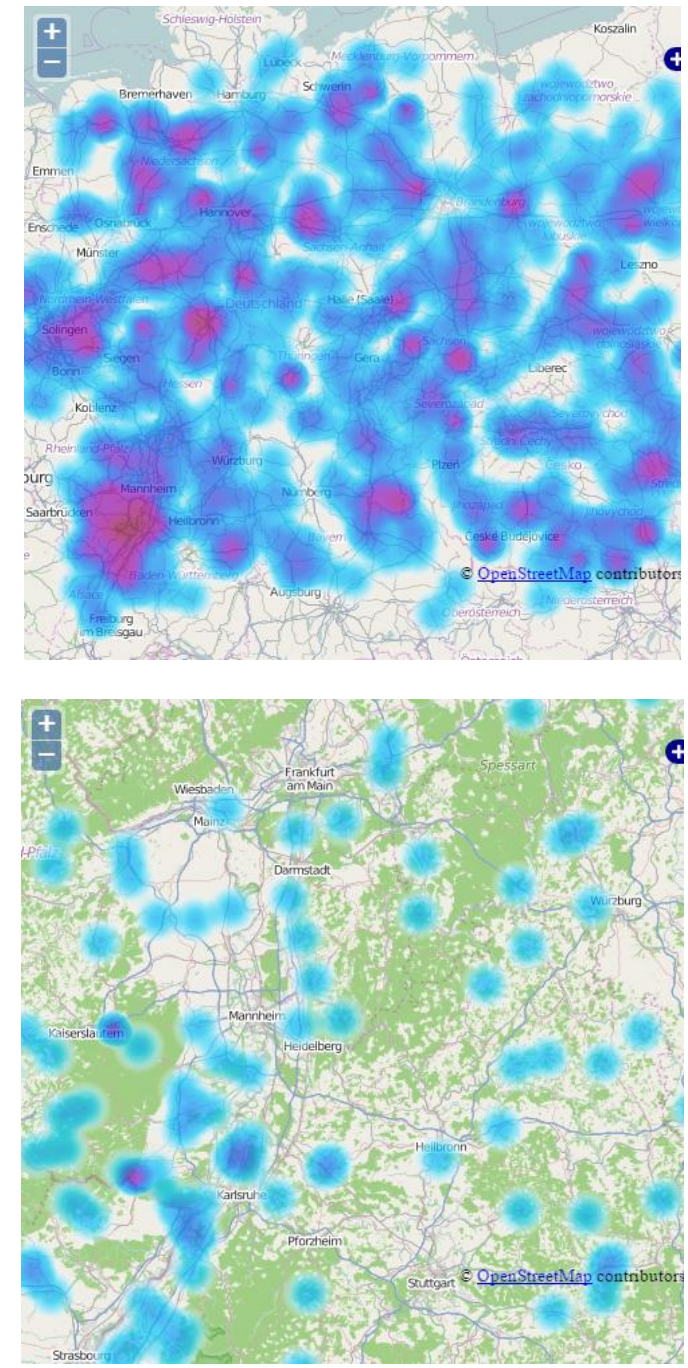

Fig. 5. Heat Map

The average values of voltage and current are calculated using the following formulas:

$$
\bar{V}_{i}=\frac{\sum_{t=t_{1}}^{t_{2}} V_{i t}}{n}
$$

Where $\bar{V}_{i}$ is the average voltage for the $i$-node of the electric network, $t_{1}$ and $t_{2}$ are the initial and final moments in time respectively. $V_{i t}$ is the voltage at the moment of time, and $n$ is the number of entries between $t_{1}$ and $t_{2}$.

\section{Color Map}

To observe the power-quality of different areas the Color Map can be used. The averaged value of the actual voltages is shown in the areas with different, user defined colors (Fig. 6). The borders of the areas are stored in the database and can be added by the user. The Color map shows the average voltage value for different regions. The even-odd algorithm is used to define if a point is inside the polygon of an area. The algorithm tests how many times a ray, starting from the point and going in any fixed direction, intersects the edges of the polygon. If the point in question is not on the boundary of the polygon, the number of intersections is an even number if the point is outside, and it is odd if inside.

The following pictures (Fig. 6) show the effect of two purpose of use. The area in the first picture is defined by user and areas in the second picture are boundary of states of Germany.
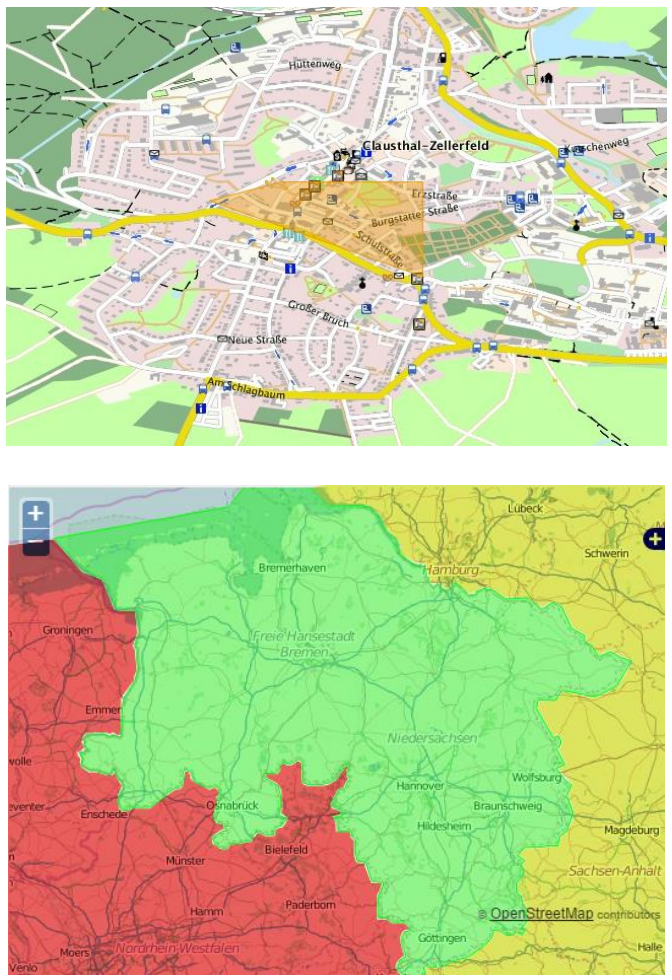

Fig. 6. Color Map

The average values of voltage and current are calculated using the following formulas:

$$
\bar{V}_{i}=\frac{\sum_{t=t_{m}}^{t_{s}} V_{i t}}{n}
$$

Where $\bar{V}_{i}$ is average voltage for the i-node of the electric network, $t_{s}$ and $t_{m}$ are the initial and final moments in time respectively. $V_{i t}$ is the voltage at the moment of time, and $\mathrm{n}$ is the number of entries between $t_{s}$ and $t_{m}$. 
Then, the average voltage of the area is calculated using the formula:

$$
\overline{\boldsymbol{V}}_{\boldsymbol{a}}=\frac{\sum \overline{\boldsymbol{V}}_{\boldsymbol{i}}}{\boldsymbol{n}_{v}}
$$

Where $n_{v}$ is the number of electric network nodes in area $\bar{V}_{a}$ and $\sum \bar{V}_{i}$ is the sum of voltages at nodes.

\section{Implementation}

This system was implemented using the Java Jumping Framework. The software architecture and basic settings are generated by Spring Roo. Spring Roo is a tool, in which Java applications can be created quickly. As a repository, MySQL is used in this prototype in the persistence layer. If the capacity of MySQL is not sufficient, it is possible to migrate to another database without high expenditure. Presentation of the information on the map and receiving of data from the measurement device is done using Java MVC Framework in User Interface implemented. Java MVC uses the MVC architecture, which is already introduced in chapter 4. JSON (JavaScript Object notation), a format for dataexchange, is used as a communication protocol in this project. JSON uses the data structured which is supported by many programming languages. Many technologies can be used to display the basic land map, like Google Maps, OpenStreetMap etc. To facilitate its development in this project the open resource technology OpenStreetMap was selected. The open-source framework OpenLayers has been chosen to show the power-quality information for Point Map and Color map. This framework has a sufficient representation method for our s. To present the heat map the Heatmaplayer.js library for OpenLayers framework is used. The time chart is presented using the Chart.js library.

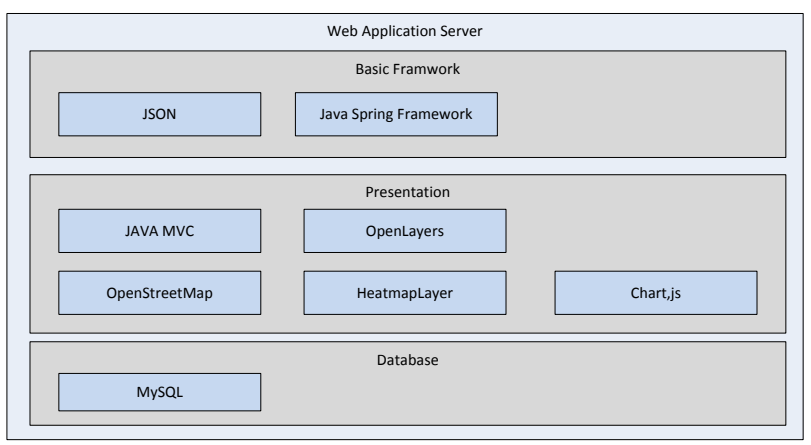

Fig.7. Used technique for developing web application server

\section{Future work}

In this work basic functions are designed to make the analysis easier and are prototype-like. Some functions are made semiautomatic, for example animation of the Heatmap. In future, this functions can extend into full automatic.

\section{Conclusion}

Using the tool, which is presented in this paper, it is possible to analyze the electricity network and easily develop the optimization algorithms for charging of electric vehicles. The charging process of electric cars can be influenced with the developed algorithms in this system, so that the network is stabilized during charging.

\section{Acknowledgement}

This work is part of the project "Charging in Smart Grid", financed by Federal Ministry for Economic Affairs and Energy.

\section{References}

[1] R. Hesse, Virtuelle Synchronmaschine, Dissertation, IEE, TU Clausthal, 2007.

[2] Y. Chen, R. Hesse, D. Turschner, H.-P. Beck, Improving the Grid Power Quality using Virtual Synchronous Machines, International Conference on Power Engineering, Energy and Electrical Drives (PowerEng 2011), Torremolinos (Spain), 2011

[3] Y. Chen, R. Hesse, D. Turschner, H.-P. Beck, Comparison of methods for implementing virtual synchronous machine on inverters, International Conference on Renewable Energies and Power Quality (ICREPQ'12), Santiago de Compostela (Spain), 2012

[4] Power Standards Lab. PQube electric power measurement instrument. http://www.powerstandards.com/PQube.php, 2013.

[5] J. Goll, J. Dausmann, Architektur- und Entwurfsmuster der Softwaretechnik, Springer Vieweg, Wiesbaden (2013). 